\title{
summary
}

\section{Penicillin does not provide effective pain relief for untreated irreversible pulpitis}

Nagle $D$, Reader A, Beck M, Weaver J. Effect of systemic penicillin on pain in untreated irreversible pulpitis. Oral Surg Oral Med Oral Pathol Oral Radiol Endod 2000; 90:636-640

\section{Question: Does systemic penicillin improve pain control in people who have irreversible pulpitis?}

Objective To determine the effect of penicillin on pain in untreated teeth diagnosed as having irreversible pulpitis.

Design Double-blind randomised controlled trial.

Intervention Forty emergency patients who had a clinical diagnosis of an irreversible pulpitis randomly received a 7-day oral dose (500 $\mathrm{mg}$ every $6 \mathrm{~h}$ ) of either penicillin or placebo in a double-blind manner. No endodontic treatment was performed until 7 days later. Each patient also received ibuprofen and acetaminophen with codeine $(30 \mathrm{mg})$. All patients completed the trial ( $0 \%$ drop-out). Patients were advised to use acetaminophen with codeine only if ibuprofen did not relieve pain.

Outcome measures Patient-assessed pain, percussion pain, and number and type of pain medication taken. At 7 days teeth were pulp-tested and the presence or absence of vital tissue confirmed by clinicians on endodontic access.

Results Penicillin did not significantly $(P>0.05)$ reduce pain, percussion pain or the number of analgesics taken by patients who had untreated irreversible pulpitis. The majority of patients with untreated irreversible pulpitis had significant pain and required analgesics to manage this pain. Three quarters of the teeth tested in the penicillin group, and $80 \%$ of the teeth tested in placebo group, were vital. None of the teeth was cariously exposed clinically.

Conclusions Penicillin should not be prescribed for untreated irreversible pulpitis because penicillin is ineffective for pain relief. Many teeth with untreated irreversible pulpitis remain symptomatic for at least a week.

Evidence-Based Dentistry (2002) 3, 72. doi:10.1038/ sj.ebd.6400114

Address for reprints: Al Reader, Graduate Endodontics College of Dentistry, PO Box 182357, Ohio State University, 305W 12th Avenue, Columbus OH 43218-2337, USA.

\section{Commentary}

Patients may have to make nonscheduled dental appointments as a result of pain arising from the dental pulp or related structures. Currently, pulpectomy or pulpotomy are believed to be the most predictable methods of relieving pain from irreversible pulpitis. ${ }^{1}$ Nevertheless, antibiotics are frequently prescribed to treat endodontic emergencies. This management modality has received much attention but little objective research had been conducted. ${ }^{2}$ Therapeutic concentrations of systemic antibiotics may reach the dental pulp ${ }^{3}$ and this study aimed to investigate whether this was of clinical value.

The administration of penicillin did not significantly reduce pain, pain on percussion or the number of analgesics taken by people who had irreversible pulpitis. The authors comment that bacteria are important in the pathogenesis of irreversible pulpitis caused by caries, but pulps of teeth without clinical exposures contain no demonstrable bacteria. This absence of bacteria and the origin of pulpal pain being mainly associated with the inflammatory process explain why antibiotics have no useful effect.

Penicillin supplementation has also been shown to have no demonstrable benefit in resolving the localised acute apical abscess. ${ }^{2}$ Patients who had localised periapical pain or swelling generally recovered quickly with appropriate local conventional endodontic treatment.

The existing evidence suggests that the use of antibiotics does not help in the pain relief of irreversible pulpitis. Pulp extirpation and conventional root canal therapy or tooth extraction remain the most predictable ways of relieving pain.

1. Oguntebi BR, DeSchepper EJ, Taylor TS, White $\mathrm{CL}$, Pink FE. Postoperative pain incidence related to the type of emergency treatment of symptomatic pulpitis. Oral Surg Oral Med Oral Pathol 1992; 73:479843.

2. Fouad AF, Rivera EM, Walton RE. Penicillin as a supplement in resolving the localized acute apical abscess. Oral Surg Oral Med Oral Pathol Oral Radiol Endod 1996; 81:590-595.

3. Haldi J, John K. Sulfanilamide and penicillin in the pulp fluid of the dog following administration of these compounds. J Dent Res 1965; 44:1386-1388.

Philip Lumley School of Dentistry, University of Birmingham, Birmingham, UK 